\title{
Acceptance and use of a smartphone application in cirrhosis
}

\author{
Jeremy Louissaint ${ }^{1}$ (D) | Anna S. Lok ${ }^{1} \mid$ Brett E. Fortune ${ }^{2}$ | Elliot B. Tapper ${ }^{1,3}$
}

\author{
${ }^{1}$ Division of Gastroenterology and \\ Hepatology, University of Michigan, Ann \\ Arbor, MI, USA \\ ${ }^{2}$ Division of Gastroenterology and \\ Hepatology, Weill Cornell Medical College, \\ New York, NY, USA \\ ${ }^{3}$ Gastroenterology Section, VA Ann Arbor \\ Healthcare System, Ann Arbor, MI, USA \\ Correspondence \\ Jeremy Louissaint, 3912 Taubman, SPC \\ 53621500 E Medical Center Dr Ann Arbor, \\ MI 48109, USA. \\ Email: jelouiss@med.umich.edu

\section{Funding information} \\ Jeremy Louissaint is supported by \\ T32DK062708. Elliot B. Tapper receives \\ funding from the National Institutes of \\ Health through (K23DK117055). \\ Handling editor: Luca Valenti
}

\begin{abstract}
Background and Aims: The development of cirrhosis-related smartphone applications for remote monitoring is increasing. Whether patients with cirrhosis will welcome such new technology, however, is uncertain.

Methods: We prospectively enrolled patients with cirrhosis $(\mathrm{N}=102)$ to determine predictors of acceptance and utilization of a smartphone application for cirrhosis management using a 12-item Technology Acceptance Model (TAM) survey. Patients were then shown the EncephalApp (C) and evaluated for their willingness to download and use the application.

Results: Patients had a median age of 61.3 years and $63.7 \%$ had a history of hepatic decompensation. Intention to use the hypothetical application was associated with perceived usefulness ( $\beta$ : $0.4,95 \% \mathrm{Cl}: 0.3-0.5)$ and the presence of a caregiver ( $\beta: 1.1,95 \% \mathrm{Cl}: 0.2-2.0$ ). Of the eligible participants, $71 \%$ agreed to download the EncephalApp (C) and the decision was influenced by computer anxiety, behavioural intent, caregiver presence and disease state factors. Actual usage was $32 \%$ and not associated with baseline characteristics or the technology acceptance model.

Conclusions: Patient acceptance of smartphone applications for the management of cirrhosis is high and related to their attitudes towards technology and the presence of a caregiver. However, usage was low. Future research must employ behavioural interventions to optimize uptake and utilization of remote monitoring technology.
\end{abstract}

\section{KEYWORDS}

cirrhosis, smartphone application, technology acceptance model, telehealth

\section{1 | INTRODUCTION}

The outpatient management of decompensated cirrhosis is complex and multifaceted. ${ }^{1}$ For ascites alone, high-quality care depends on the adherence to dietary sodium restriction, weight tracking, timely and careful diuretic adjustments, electrolyte and renal function monitoring, and the coordination of large volume paracenteses when needed. ${ }^{2,3}$ When outpatient monitoring fails, the risk of an

Abbreviations: CANX, computer anxiety; $\mathrm{Cl}$, confidence interval; IQR, interquartile range; MELD-Na, Model for End-Stage Liver Disease Sodium; OR, odds ratio; PEOU, perceived ease of use; PU, perceived usefulness; SD, standard deviation; TAM, Technology Acceptance Model; USD, United States Dollars. otherwise preventable admission increases, carrying with it the risk of readmission. ${ }^{4,5}$ Hospitalization, in turn, diminishes health-related quality of life and places an exceptional burden on patients and their caregivers in addition to healthcare costs and risks of hospital-acquired infections. ${ }^{6,7}$ Office-based visits are essential but may be too infrequent to coordinate care and capture decompensating events at a stage where successful intervention can prevent hospitalization. ${ }^{8}$ For this reason, interest in the use of telehealth as an adjunct to outpatient follow-up is on the rise.

Multiple platforms for telehealth have been evaluated for persons with cirrhosis. In a prospective trial from our centre, automated interactive voice calls were able to effectively identify individuals 
most at risk for hospitalization based on symptoms such as weakness and rapid weight gain. ${ }^{9}$ Elsewhere, live telephone-based communications between healthcare workers and patients in the post-discharge period were associated with improved 6-month survival in cirrhosis. ${ }^{10}$ Naturally, as the vast majority of people now own smartphones, there has been a shift towards the development of smartphone applications to provide a more continuous and easily expandable method of outpatient disease monitoring. ${ }^{11-13}$ As illustrated by Ganapathy et al, a smartphone application provided to persons with cirrhosis and their caregivers that was capable of monitoring psychometric performance was able to identify and alert the care team to changes in a patient's cognition, facilitating early intervention. ${ }^{14}$ Nonetheless, there are currently minimal data on patient acceptance of smartphone applications to track their disease. Knowledge of factors associated with a patient's acceptance and use of technology to manage their liver disease is important in guiding the development of these novel tools. ${ }^{15}$ In the current study, we measured overall technology acceptance in a cohort of patients with cirrhosis using a modified technology acceptance model survey and explored predictors of its uptake and usage.

\section{2 | METHODS}

\subsection{Patient selection}

Using convenience sampling, all patients age $>18$ years with cirrhosis, presenting for an outpatient hepatology or liver transplant clinic visit at our hospital between 10/2019 and 1/2020, were eligible for inclusion in the study. No patients were recruited during the last week of 11/2019 or at all during 12/2019 to avoid participation during the holidays. The diagnosis of cirrhosis must have been documented in a previous clinic encounter by the patient's hepatologist and supported by clinical, laboratory, imaging, elastography or biopsy data. Manual chart review was also performed to determine cirrhosis stage (compensated or decompensated), history of decompensating events (variceal bleeding, hepatic encephalopathy, ascites, hepatorenal syndrome, hepatic hydrothorax and spontaneous bacterial peritonitis), disease severity (Model for End-Stage Liver Disease Sodium, MELD-Na) and cirrhosis-related medications. Additionally, we collected information regarding a patient's zip code (to determine median household income by zip code), the presence of an activated patient portal, and the presence and type of smartphone they owned. Patients were excluded if they had a history of a liver transplantation, were not English speaking or could not read English, or had significant mental deficits at the time of enrolment (dementia, West Haven grade 2 or higher hepatic encephalopathy).

\section{2 | Survey design and administration}

The technology acceptance model (TAM) is a theoretical framework that seeks to explain technology acceptance and use. ${ }^{16}$ TAM

\section{Key points}

- The care of patients with cirrhosis is complex and requires frequent monitoring.

- There is increasing interest in augmenting ambulatory monitoring through the use of smartphone applications.

- However, an understanding of factors associated with patient acceptance of these technologies is lacking.

- Here, we show that principles of the technology acceptance model (perceived usefulness, perceived ease of use and computer anxiety) as well as patient baseline characteristics can easily identify potential adopters of a cirrhosis-related smartphone application.

assesses how a potential user perceives the usefulness (perceived usefulness, PU) and ease of use (perceived ease of use, PEOU) of a new technology, and how those factors impact that technology's uptake and use. ${ }^{15,16}$ Combined, perceived usefulness and perceived ease of use account for $40 \%$ of an individual's intention to use (behavioural intent) and actual usage of a novel technology. ${ }^{17}$ One important contributor to perceived ease of use is emotional in nature and termed Computer Anxiety (CANX), or the extent to which one is fearful or averse to technology. ${ }^{18}$ While further research has expanded on several additional key factors that influence both perceived usefulness and perceived ease of use, the TAM model in the current study included items related to computer anxiety, perceived ease of use, perceived usefulness and behavioural intent. ${ }^{15}$

Prior to administering the survey, patients were asked to read a short prompt about a hypothetical interactive phone application ('Cirrhosis Health Application') designed to help in cirrhosis care (Figure S1). The statement described the potential utility of the phone application in the management of ascites, hepatic encephalopathy, medication adherence and overall disease state, similar to a previously studied mobile application (the Patient Buddy application). ${ }^{14}$ We then administered a modified 12 -item TAM survey (Likert scale from 1 'definitely disagree' to 7 'definitely agree'), with higher scores indicating higher technology acceptance (Figure S2). It is conventional to adapt the questions of the TAM survey to reflect the specific technology under evaluation. ${ }^{19,20}$ To ensure face validity while modifying the questions to focus on the Cirrhosis Health Application, we tested and revised the modified questions with physicians and health services researchers not associated with the study. Computer anxiety questions were negatively scaled so that higher scores indicated less comfort with technology. We also assessed for the presence of an in-home informal caregiver, defined here as a family member or friend who could, if needed, assist in basic activities of daily living. As a freely available comprehensive cirrhosis-related health application does not yet exist, we used as a test-case the canonical cirrhosis app: the EncephalApp 
Stroop Test $\odot$, which is a free, well studied and clinically useful point-of-care smartphone application designed and able to detect no, covert and overt hepatic encephalopathy. ${ }^{21-25}$ Following survey completion, patients owning a smartphone and without red-green colour blindness (an exclusion criterion for the EncephalApp (C) were told the purpose of and then actively watched a member of the research team (JL) perform a short run of the EncephalApp C to ensure understanding (Figure 1). Participants were then asked if they would be agreeable to download the application onto their own smartphone with the intention of using it once or twice between 7 and 15 days after installation. Agreeable participants then downloaded the application with the help of a member of the research team (JL). Settings were adjusted to make the testing non-diagnostic as we could not control the environment in which the application would be completed and the results interpreted. The study email address was entered into the application so that after a participant completed the application, the study team would be alerted via email. After 18 days post-enrolment, we attempted to contact those who downloaded but did not complete the EncephalApp $\subset$ to assess for any barriers that prevented use of the application. The study received approval from the University of Michigan Institutional Review Board and all study participants provided written consent.

\section{3 | Statistical analysis}

Descriptive data were presented as number (percent) for categorical data and mean (standard deviation, SD) or median (interquartile range, IQR) for continuous data. We sought to characterize factors (demographic and clinical characteristics, perceived usefulness, perceived ease of use, computer anxiety) associated with higher behavioural intent (the statistical mean of questions 9 and 10) to use the hypothetical Cirrhosis Health Application. Correlations between TAM constructs were analysed using Pearson's correlation coefficient. Linear regression was used to identify predictors of behavioural intent and logistic regression was used to determine if demographic and clinical characteristics as well as TAM constructs could explain a patient's decision to download and use the EncephalApp (. These analyses were performed initially using univariable logistic regression. Given multiple predictor variables and multicollinearity, penalized regression using Lasso regression was performed for variable selection. ${ }^{26}$ Non-zeroed variables from the Lasso regression were then analysed in a multivariable logistic regression model. Significance was determined using 95\% confidence intervals $(95 \% \mathrm{Cl})$, and $P<.05$. We hypothesized that behavioural intent would increase by $20 \%$ in the presence of decompensated cirrhosis. Using mean and standard deviation behavioural intent values

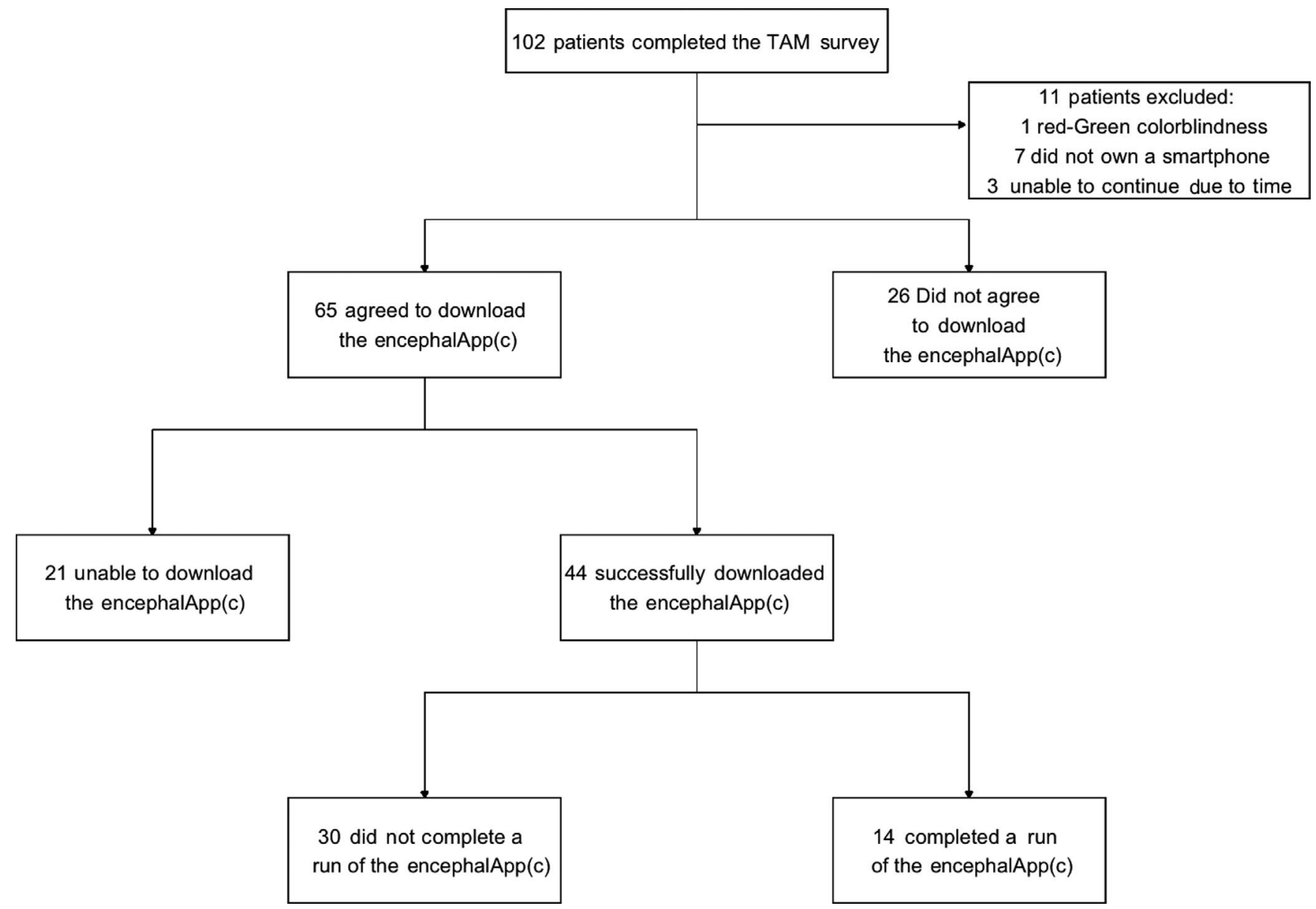

FIGURE 1 Flow diagram of patient acceptance and use of the EncephalApp ( 
from Venkatesh et $\mathrm{al}^{15}$ and power of 0.8 , we required a sample size of at least 68 patients. Data were collected using REDCap. We performed the statistical analysis using RStudio. ${ }^{27}$

\section{3 | RESULTS}

A total of 140 patients were approached for inclusion in the study and a total of 102 (73\%) patients agreed to complete the survey. Participants had a median age of 61.3 years, were predominantly white (90.2\%) and $56.9 \%$ were men (Table 1). Cirrhosis was most commonly related to alcohol use disorder (37.3\%) and non-alcoholic fatty liver disease $(30.4 \%)$. A history of decompensation was present in $63.7 \%$ of participants at the time of enrolment. A history of ascites and hepatic encephalopathy was present in $48(47.1 \%)$ and 40 (39.2\%) patients, respectively. The median MELD-Na was 10. Smartphone ownership was $93.1 \%$.

TABLE 1 Baseline characteristics

\begin{tabular}{|c|c|}
\hline Demographics & $N=102(\%)$ \\
\hline Age, median & $\begin{array}{l}61.30 \text { (range: } \\
24-90 \text { ) }\end{array}$ \\
\hline Male & $58(56.9 \%)$ \\
\hline \multicolumn{2}{|l|}{ Race } \\
\hline White/Caucasian & $92(90.2)$ \\
\hline Black/African American & $9(8.8)$ \\
\hline Other/unknown & $1(1.0)$ \\
\hline Median household income by zip code (USD) & 58680 \\
\hline \multicolumn{2}{|l|}{ Cirrhosis aetiologies $^{a}$} \\
\hline Non-alcoholic fatty liver disease & $31(30.4)$ \\
\hline Alcohol & 38 (37.3) \\
\hline Hepatitis $C$ virus & $18(17.6)$ \\
\hline Hepatitis $B$ virus & $2(2.0)$ \\
\hline Other & $27(26.5)$ \\
\hline \multicolumn{2}{|l|}{ Cirrhosis complication(s) $^{a}$} \\
\hline Ascites & $48(47.1)$ \\
\hline Hepatic encephalopathy & $40(39.2)$ \\
\hline Variceal haemorrhage & $14(13.7)$ \\
\hline Spontaneous bacterial peritonitis & $11(10.8)$ \\
\hline Hepatic hydrothorax & $8(7.8)$ \\
\hline Hepatorenal syndrome & $5(4.9)$ \\
\hline None & $36(36.3)$ \\
\hline Median MELD-Na (IQR) & $10.0(8.0-15.8)$ \\
\hline Active patient portal & $71(69.6)$ \\
\hline Caregiver & $76(74.5)$ \\
\hline
\end{tabular}

Abbreviations: USD, United States dollars; MELD-Na, model for endstage liver disease sodium; IQR, interquartile range. a May have $>1$ aetiology or complication of cirrhosis

\section{1 | Behavioural intent to use the hypothetical cirrhosis health app}

Mean survey item scores for perceived usefulness, perceived ease of use and the absolute value of computer anxiety were between 5 and 5.2 out of 7 (SD 1.2-1.8) (Table 2). Behavioural intent to use the hypothetical cirrhosis health application was significantly correlated with perceived usefulness ( $r=.77,95 \% \mathrm{Cl}$ : 0.67-0.84), perceived ease of use $(r=.65,95 \% \mathrm{Cl}: 0.52-0.75)$ and computer anxiety ( $r=-.54,95 \% \mathrm{Cl}:-0.66$ to -0.38$)$. Combined, these variables explained $61 \%$ of the variance in behavioural intent to use the Cirrhosis Health Application (adjusted $R^{2}=.61$ ).

Univariable linear regression for predictors of behavioural intent is illustrated in Table 3. Variable selection using Lasso regression revealed age, median household income by zip-code, perceived usefulness, perceived ease of use, computer anxiety, the presence of a caregiver and the presence of an ascites medication as important predictors of behavioural intent. Together, these variables explained $64 \%$ of the variance in behavioural intent (adjusted $R^{2}=.64$ ). On multiple logistic regression, significant predictors of behavioural intent included perceived usefulness ( $\beta=0.4,95 \% \mathrm{Cl}$ : $0.3-0.5$ ) and caregiver presence ( $\beta=1.1,95 \% \mathrm{Cl}$ : 0.2-2.0).

Analysis of characteristics associated with the antecedents of behavioural intent (perceived usefulness, perceived ease of use and computer anxiety) revealed that the presence of a caregiver was associated with higher perceived usefulness ( $\beta=2.7,95 \% \mathrm{Cl}$ : 0.5-4.8), perceived ease of use ( $\beta=3.8,95 \% \mathrm{Cl}$ : 1.8-5.9) and lower computer anxiety $(\beta=-2.5,95 \% \mathrm{Cl}:-4.0$ to -0.9$)$. For every 1-year increase in age, there was both a 0.09 increase in computer anxiety $(95 \% \mathrm{Cl}$ : 0.04-0.1) and decrease in perceived ease of use $(95 \% \mathrm{Cl}$ : -0.2 to -0.01). Neither MELD-Na nor median household income by zip code was correlated with perceived usefulness, perceived ease of use or computer anxiety.

\section{2 | Factors associated with agreement to download the EncephalApp $\subset$}

Of the 102 participants who completed the TAM survey, 11 (10.8\%) were excluded ( 1 for red/green colour blindness, 7 due to not owning a smartphone and 3 who were unable to continue due to time

TAB LE 2 TAM domain scores in the study population $(\mathrm{N}=102)$

\begin{tabular}{|c|c|c|c|}
\hline Domain & $\begin{array}{l}\text { Question } \\
\text { numbers }\end{array}$ & Mean (SD) & $\begin{array}{l}\text { Item mean } \\
\text { (SD) }\end{array}$ \\
\hline Perceived usefulness (PU) & $1,3,5,7$ & $20.8(5.0)$ & $5.2(1.2)$ \\
\hline $\begin{array}{l}\text { Perceived ease of use } \\
\text { (PEOU) }\end{array}$ & $2,4,6,8$ & $20.0(4.9)$ & $5.0(1.2)$ \\
\hline $\mathrm{PU}+\mathrm{PEOU}$ & & $40.7(9.1)$ & \\
\hline Computer anxiety & 11,12 & $-10.2(3.6)$ & $-5.1(1.8)$ \\
\hline Behavioural intent & 9,10 & $10.2(3.1)$ & $5.1(1.6)$ \\
\hline
\end{tabular}

Abbreviations: TAM, technology acceptance model; SD, standard deviation. 
TABLE 3 Variables associated with behavioural intent to use the cirrhosis health application

\begin{tabular}{|c|c|c|}
\hline$N=102$ & $\begin{array}{l}\text { Univariable: } \\
\beta \text { coefficient } \\
(95 \% \mathrm{Cl})\end{array}$ & $\begin{array}{l}\text { Multivariable: } \\
\beta \text { coefficient }(95 \% \mathrm{Cl})\end{array}$ \\
\hline Age (years) & $\begin{array}{l}-0.06(-0.1 \text { to } \\
-0.02)\end{array}$ & $-0.02(-0.05$ to 0.01$)$ \\
\hline Sex (male) & $-0.7(-1.9$ to 0.6$)$ & \\
\hline MELD-Na & $0.04(-0.1$ to 0.2$)$ & \\
\hline $\begin{array}{l}\text { Median household } \\
\text { income by zip code } \\
\text { (USD) }\end{array}$ & $0.07(-0.2$ to 0.4$)$ & $-0.07(-0.3$ to 0.1$)$ \\
\hline Decompensated & $0.4(-0.8$ to 1.7$)$ & \\
\hline History of ascites & 0.5 (-0.7 to 1.7$)$ & \\
\hline $\begin{array}{l}\text { History of hepatic } \\
\text { encephalopathy }\end{array}$ & 0.6 (-0.7 to 1.8$)$ & \\
\hline $\begin{array}{l}\text { No cirrhosis } \\
\text { medication }\end{array}$ & $-0.6(-1.8$ to 0.7$)$ & \\
\hline $\begin{array}{l}\text { Hepatic } \\
\text { encephalopathy } \\
\text { medication }\end{array}$ & $0.8(-0.4$ to 2.1$)$ & \\
\hline Ascites medication & $0.7(-0.5$ to 2.0$)$ & $0.7(-0.07$ to 1.4$)$ \\
\hline Caregiver presence & $2.6(1.3-3.9)$ & $1.1(0.2-2.0)$ \\
\hline Active portal account & $1.6(0.3-2.9)$ & \\
\hline Perceived usefulness & $0.5(0.4-0.6)$ & $0.4(0.3-0.5)$ \\
\hline Perceived ease of use & $0.4(0.3-0.5)$ & $0.06(-0.06$ to 0.2$)$ \\
\hline Computer anxiety & $\begin{array}{l}-0.5(-0.6 \text { to } \\
-0.3)\end{array}$ & $-0.09(-0.2$ to 0.05$)$ \\
\hline
\end{tabular}

Abbreviations: $\mathrm{Cl}$, confidence interval; MELD-Na, model for end-stage liver disease sodium; USD, United States dollars.

constraints). The remaining 91 (89.2\%) patients were shown the EncephalApp $\odot$ and 65 (71.4\%) patients expressed willingness to download the EncephalAppœ. On univariable logistic regression, agreement to download the application was increased in the presence of an informal caregiver (OR: 4.03, 95\% Cl: 1.44-11.29), a history of hepatic decompensation (OR: $3.07,95 \% \mathrm{Cl}: 1.20-7.85$ ), a history of ascites (OR: $3.44,95 \% \mathrm{Cl}: 1.22-9.67)$ or the presence of any cirrhosis-related medication (OR: $2.56,95 \% \mathrm{Cl}: 1.01-6.52$ ), in particular, the presence of an ascites-related medication (OR: 3.04, 95\% Cl: 1.08-8.54) (Table 4). Additionally, perceived usefulness (OR: 1.07, $95 \% \mathrm{Cl}: 1.05-1.30$ ), perceived ease of use (OR: $1.19,95 \% \mathrm{Cl}$ : 1.06-1.33), computer anxiety (OR: $0.75,95 \% \mathrm{Cl}: 0.64-0.87)$ and behavioural intent (OR: $1.35,95 \% \mathrm{Cl}: 1.12-1.62)$ scores were all significantly associated with agreement to download the EncephalApp@). Variable selection after Lasso regression identified computer anxiety, behavioural intent, presence of a caregiver, presence of an ascites medication, hepatic decompensation and a history of ascites as important model covariates. Computer anxiety was the only variable significantly associated with willingness to download the EncephalApp ( ) on multivariable logistic regression (OR: 0.78, 95\% Cl: 0.65-0.93).
TABLE 4 Analysis of factors associated with willingness to download the EncephalApp (C)

\begin{tabular}{|c|c|c|}
\hline \multirow{2}{*}{\multicolumn{3}{|c|}{$\begin{array}{l}\mathrm{N}=91 \\
\text { Agreed to Download }(\mathrm{N}=65)\end{array}$}} \\
\hline & & \\
\hline Did not Agree to & Univariable: & Multivariable: \\
\hline Downloaders $(\mathrm{N}=26)$ & OR (95\% Cl) & OR (95\% CI) \\
\hline Age (years) & $0.98(0.95-1.02)$ & \\
\hline Sex (male) & $0.69(0.27-1.73)$ & \\
\hline $\begin{array}{l}\text { Median household income by } \\
\text { zip code (USD) }\end{array}$ & $1.08(0.84-1.38)$ & \\
\hline Perceived usefulness (PU) & $1.17(1.05-1.30)$ & \\
\hline Perceived ease of use (PEOU) & $1.19(1.06-1.33)$ & \\
\hline Computer anxiety (CANX) & $0.75(0.64-0.87)$ & $0.78(0.65-0.93)$ \\
\hline Behavioural intent (BI) & $1.35(1.12-1.62)$ & $1.11(0.89-1.38)$ \\
\hline MELD-Na & $1.05(0.96-1.15)$ & \\
\hline Caregiver presence & $4.03(1.44-11.29)$ & $2.31(0.68-7.83)$ \\
\hline Active portal account & $1.62(0.61-4.34)$ & \\
\hline Decompensated & $3.07(1.20-7.85)$ & $1.39(0.30-6.38)$ \\
\hline Ascites & $3.44(1.22-9.67)$ & $1.56(0.26-9.27)$ \\
\hline Hepatic encephalopathy & $2.69(0.95-7.56)$ & \\
\hline $\begin{array}{l}\text { Taking any cirrhosis-related } \\
\text { medication }\end{array}$ & $2.56(1.01-6.52)$ & \\
\hline Ascites medication & $3.04(1.08-8.54)$ & $\begin{array}{l}2.05 \\
(0.34-12.48)\end{array}$ \\
\hline $\begin{array}{l}\text { Hepatic encephalopathy } \\
\text { medication }\end{array}$ & $2.05(0.76-5.56)$ & \\
\hline
\end{tabular}

Abbreviations: $\mathrm{Cl}$, confidence interval; USD, United States dollars; MELD-Na, model for end-stage liver disease sodium.

\subsection{Use of the EncephalApp@ smartphone application}

Overall, 21 (32.3\%) of 65 patients who expressed willingness to download the application were not able to successfully do so. Six patients were found to not have their phone with them, 4 were not able to access the Internet at the time of enrolment, 9 did not know their smartphone application store log-in information and 2 patients did not have enough storage on their device. Of the remaining $44(67.7 \%)$ patients who agreed to and successfully downloaded the EncephalApp $\odot$, we received email evidence of its use from 14 (31.8\%) patients on at least one occasion between day 7 and 15 after enrolment. Neither TAM constructs nor baseline characteristics could explain the likelihood of application usage (Table S1).

\section{4 | Barriers to EncephalApp@ use}

We attempted to contact the 30 patients for whom we did not receive an application-generated email showing proof of application completion to assess for any barriers to the application's use. Four (13.3\%) patients were hospitalized in the 16 days after they downloaded the application. Of the remaining 26 participants, we were 
able to successfully contact $13(50 \%)$ of them to assess for barriers to use of the application. Ten (76.9\%) simply forgot to complete the application, 1 (7.7\%) noted difficulty running the application and 2 (15.4\%) stated the application was indeed completed which also suggested a technical issue occurred.

\section{4 | DISCUSSION}

Early identification of cirrhosis complications in the ambulatory setting is necessary to improve the quality of care provided to patients with chronic liver disease. Mobile telehealth tools can be employed to enhance outpatient monitoring beyond what can be provided with traditional outpatient visits alone. ${ }^{13}$ However, a lack of understanding about the factors that would lead to technology use in this population would simply result in the creation of underused and financially expensive interventions. ${ }^{15,18,28}$ Our results show that those most likely to download a cirrhosis-related smartphone application are readily identified using principles from the technology acceptance model (perceived usefulness, perceived ease of use and computer anxiety), the presence of cirrhosis complications and medications, and the presence of an informal caregiver. Nonetheless, our results also highlight the barriers that must be overcome to achieve a meaningful level of usage. Even among patients who downloaded a mobile telehealth application in clinic, we observed low levels of after-visit engagement with the technology. Our data therefore show that even for patients who are comfortable with technology, no smartphone application-based monitoring programme can be successfully deployed without additional coaching or behavioural interventions. This study offers several lessons to extend our knowledge of how patients with cirrhosis interact with technology.

\subsection{Acceptance of the hypothetical cirrhosis health application}

We found that nearly $70 \%$ of patients perceived the hypothetical Cirrhosis Health Application as useful (mean perceived usefulness score of 5 or higher). This favourable result likely reflected two study design decisions that should serve as a guide for how future applications are designed and introduced to patients. First, the information provided about the application was simple, positive and meant to help patients easily identify if there was a function of the application that applied to their care. We did not assume patients understood the drivers of their complications as patient knowledge of cirrhosisrelated care and complications ranges from $25 \%$ to $53 \% .{ }^{29,30}$ Second, the information provided likely highlighted to patients the potential future complications they could face; therefore, how the application could be of use in the future. Both of these design factors likely improved the technology's relevance to the end-user and contributed to perceived usefulness. ${ }^{15}$

The fact that patients were unable to interact with either the hypothetical Cirrhosis Health Application or the EncephalApp๔ prior to answering the TAM survey questions was a strength of the study. When measured prior to an interaction with a new system, perceived ease of use values reflect a patient's general confidence in their use of technology rather than system-specific factors. ${ }^{31}$ Therefore, our finding of high perceived ease of use leading to increased behavioural intent to use the hypothetical Cirrhosis Health Application is generalizable, at least in our population, to other cirrhosis-related smartphone applications designed to monitor medication and dietary adherence, weight and psychometric changes.

\subsection{Antecedents of technology acceptance and adoption}

In the current study, we expand the current understanding of technology acceptance and adoption in cirrhosis. The available literature has revealed promising results from studies employing tablets and mobile devices in cirrhosis care. For example, providing patients and caregivers with mobile devices with a pre-installed cirrhosis care application can potentially prevent hepatic encephalopathy-related readmissions. ${ }^{14}$ Bloom et al showed that administering Bluetoothenabled scales to patients with ascites resulted in successful (70\%) transmission of weights during the study period. ${ }^{32}$ Lastly, in an observational study where tablets for telemonitoring (in-home monitoring of vital signs) were provided to post-liver transplantation patients, high use was associated with lower readmissions. ${ }^{33}$ However, giving devices to everyone overlooks key adoption and use factors. Indeed, we found that patients who were very accepting of technology actually demonstrated low rates of after-visit use. Moreover, administering a novel technology indiscriminately is not practical or economically sound on a large scale.

Care teams must understand important antecedents of behavioural intent when designing and implementing novel cirrhosis-related smartphone applications. These elements include how useful a patient perceives the technology, which includes easily identifiable disease-state factors, as well as the patient's self-reported comfort with technology and the presence of a caregiver (Figure S3). All of these characteristics can be readily gathered with minimal effort during a clinic encounter. Combined, these factors explain nearly two-thirds of a patient's intention to use a cirrhosis-related mobile telehealth application, which is a critical step on the pathway to ultimate usage.

\section{3 | The impact of informal caregivers}

The presence of an informal caregiver was significantly associated with all TAM domains (perceived usefulness, perceived ease of use, computer anxiety, behavioural intent), and greatly influenced a patient's overall decision to download the EncephalApp $\odot$. The shared psychological, financial and quality-of-life disease burden seen in patients with cirrhosis and their caregivers may partly explain this finding. ${ }^{34-36}$ It is conceivable that patients with informal caregivers 
saw a perceived usefulness that extended beyond themselves and encompassed their caregiver. In fact, if an individual believes that someone close to them thinks that he/she should use the technology, then the individual's perceived usefulness of the technology is significantly increased. ${ }^{17}$ We also saw significantly less computer anxiety and more perceived ease of use scores in participants with informal caregivers. We postulate that perhaps the technological help participants believed an informal caregiver could provide increased their comfort with technology use. Our study did not explicitly involve caregivers but given low after-visit utilization, future interventions should incorporate caregivers to reinforce the use of remote monitoring technology.

\section{4 | Barriers to Encephalapp@ use}

While we were able to identify elements that increased a patient's acceptance and willingness to download a smartphone application for cirrhosis care, actual usage was modest (32\%) and the small sample size likely precluded an analysis of predictors of use. More broadly, the per cent of patients who used the application from the overall patient population was only $14 \%$. This highlights the multiple barriers to technology use in cirrhosis that must be considered in leveraging technology to improve cirrhosis care. First, technologyrelated barriers - no smartphone, difficulty accessing the Internet in clinic, unknown application store information and inadequate phone storage - accounted for a $32 \%$ decrease in the number of patients who could download the application. Second, even after successful installation, personal factors, such as forgetfulness and intervening hospitalizations, precluded use of the EncephalApp ( . Certainly, future implementations of smartphone applications should include telephone follow-up to reiterate instructions on its use, reminders for use and potentially a help-line.

\section{5 | Contextual factors/limitations}

Our data must be interpreted within the context of the study design. First, survey responses were based on opinions about the imaginary Cirrhosis Health Application, but analysis of the decision to download an application was determined based on the EncephalApp (C. Despite this, results remained consistent with higher behavioural intent to use the Cirrhosis Health Application (which included a psychometric component) being associated with a higher likelihood of downloading the EncephalApp $\odot$. Second, in informing patients that the results obtained from the EncephalApp $\odot$ would not be used to guide their management, perceived usefulness was effectively reduced. Notably, in spite of this information, $71 \%$ of eligible participants still expressed willingness to download the application. Third, the EncephalApp $\odot$ was created as a point-of-care test for detecting minimal hepatic encephalopathy in the clinical setting. ${ }^{25}$ However, in-home use of the EncephalApp (C) has been shown to be feasible and effective for detecting cognitive changes in persons with cirrhosis. ${ }^{14}$ Fourth, the study population had a relatively low median MELD-Na and was likely more clinically stable than a sample of transplant waitlisted patients. However, interventions, such as mobile applications, are likely best deployed at a stage where successful intervention can prevent hospitalization. ${ }^{8}$ Given the high rate of prior decompensation in our sample, this represents an ideal study population where the risk of future events is high but there is likely an important decompensation-free interval where individuals have time to use and become acclimated to these novel interventions. Lastly, in practice, various members of a care team would approach patients about downloading a novel smartphone technology. In the current study, the same individual recruited all participants so as to not introduce differences in recruiters as a confounding variable.

\section{5 | CONCLUSION}

In cirrhosis, overall patient acceptance of a hypothetical cirrhosisrelated smartphone application is high; though multiple barriers exist that limit actual usage. Those most agreeable to adopt these technologies can be identified based on available clinical factors and principles from the technology acceptance model. Perceived usefulness, perceived ease of use and computer anxiety, as well as the disease stage and the presence of an informal caregiver, should be of paramount importance in the successful development and implementation of mobile technologies to improve cirrhosis care.

\section{CONFLICT OF INTERESTS}

Jeremy Louissaint is the guarantor of this article. Roles-Concept: Louissaint; Analysis: Louissaint, Tapper, Lok; Data acquisition: Louissaint; Writing: Louissaint; Revision: Tapper, Lok, Fortune. Elliot

B. Tapper has served as a consultant to Norvartis and Allergan, has served on advisory boards for Mallinckrodt and Bausch Health and has received unrestricted research grants from Gilead and Valeant. Valeant is the maker of Rifaximin, a medication approved for treatment of hepatic encephalopathy. No other author has a conflict of interest.

\section{ORCID}

Jeremy Louissaint (iD https://orcid.org/0000-0003-1154-1825

\section{REFERENCES}

1. Angeli P, Bernardi M, Villanueva C, et al. EASL Clinical Practice Guidelines for the management of patients with decompensated cirrhosis. J Hepatol. 2018;69:406-460.

2. Runyon BA. Care of patients with ascites. $N$ Engl J Med. 1994;330:337-342.

3. Runyon BA; AASLD Practice Guidelines Committee. Management of adult patients with ascites due to cirrhosis: an update. Hepatol Baltim Md. 2009;49:2087-2107.

4. Tapper EB, Volk M. Strategies to reduce 30-day readmissions in patients with cirrhosis. Curr Gastroenterol Rep. 2017;19:1.

5. TapperEB, Halbert B, Mellinger J. Rates of and reasons for hospital readmissions in patients with cirrhosis: a multistate population-based cohort study. Clin Gastroenterol Hepatol. 2016;14:1181-1188. e2. 
6. Tapper EB, Kanwal F, Asrani SK, et al. Patient-reported outcomes in cirrhosis: a scoping review of the literature. Hepatology. 2018;67:2375-2383.

7. Rakoski MO, McCammon RJ, Piette JD, et al. Burden of cirrhosis on older Americans and their families: analysis of the health and retirement study. Hepatol Baltim Md. 2012;55:184-191.

8. Kanwal F, Asch SM, Kramer JR, et al. Early outpatient follow-up and 30-day outcomes in patients hospitalized with cirrhosis. Hepatol Baltim Md. 2016;64:569-581.

9. Thomson M, Volk M, Kim HM, et al. An automated telephone monitoring system to identify patients with cirrhosis at risk of re-hospitalization. Dig Dis Sci. 2015;60:3563-3569.

10. Rao BB, Sobotka A, Lopez R, et al. Outpatient telephonic transitional care after hospital discharge improves survival in cirrhotic patients. World J Hepatol. 2019;11:646-655.

11. Patel MS, Polsky D, Kennedy EH, et al. Smartphones vs wearable devices for remotely monitoring physical activity after hospital discharge: a secondary analysis of a randomized clinical trial. JAMA Netw Open. 2020;3:e1920677.

12. Gordon WJ, Landman A, Zhang H, et al. Beyond validation: getting health apps into clinical practice. Npj Digit Med. 2020;3:1-6.

13. Stotts MJ, Grischkan JA, Khungar V. Improving cirrhosis care: The potential for telemedicine and mobile health technologies. World J Gastroenterol. 2019;25:3849-3856.

14. Ganapathy D, Acharya C, Lachar J, et al. The patient buddy app can potentially prevent hepatic encephalopathy-related readmissions. Liver Int Off J Int Assoc Study Liver. 2017;37:1843-1851.

15. Venkatesh $\mathrm{V}$, Bala $\mathrm{H}$. Technology acceptance model 3 and a research agenda on interventions. Decis Sci. 2008;39:273-315.

16. Davis FD. Perceived usefulness, perceived ease of use, and user acceptance of information technology. MIS Q. 1989;13:319-340.

17. Venkatesh V, Davis FD. A theoretical extension of the technology acceptance model: four longitudinal field studies. Manag Sci. 2000;46:186-204.

18. Venkatesh V. Determinants of perceived ease of use: integrating control, intrinsic motivation, and emotion into the technology acceptance model. Inf Syst Res. 2000;11:342-365.

19. Orruño E, Gagnon MP, Asua J, et al. Evaluation of teledermatology adoption by health-care professionals using a modified Technology Acceptance Model. J Telemed Telecare. 2011;17:303-307.

20. Wade R, Cartwright C, Shaw K. Factors relating to home telehealth acceptance and usage compliance. Risk Manag Healthc Policy. 2012;5:25-33.

21. Bajaj JS, Heuman DM, Sterling RK, et al. Validation of Encephalapp, smartphone-based stroop test, for the diagnosis of covert hepatic encephalopathy. Clin Gastroenterol Hepatol Off Clin Pract J Am Gastroenterol Assoc. 2015;13:1828-1835.e1.

22. Tapper EB, Parikh ND, Waljee AK, et al. Diagnosis of minimal hepatic encephalopathy: a systematic review of point-of-care diagnostic tests. Am J Gastroenterol. 2018;113:529-538.
23. Bajaj JS. Adventures in developing an app for covert hepatic encephalopathy. Clin Transl Gastroenterol. 2017;8:e85.

24. Allampati S, Duarte-Rojo A, Thacker LR, et al. Diagnosis of minimal hepatic encephalopathy using stroop Encephalapp: a multicenter US-based, norm-based study. Am J Gastroenterol. 2016;111:78-86.

25. Bajaj JS, Thacker LR, Heuman DM, et al. The Stroop smartphone application is a short and valid method to screen for minimal hepatic encephalopathy. Hepatol Baltim Md. 2013;58:1122-1132.

26. Tibshirani R. Regression shrinkage and selection via the lasso. $J R$ Stat Soc Ser B Methodol. 1996;58:267-288.

27. Anon. RStudio | Open source \& professional software for data science teams - RStudio. https://rstudio.com/ (Accessed: January 13, 2020).

28. Lee T, Kim L. Telemedicine in gastroenterology: a value added service for patients. Clin Gastroenterol Hepatol. 2019. http://www.sciencedirect.com/ science/article/pii/S1542356519314041 (Accessed: January 30, 2020).

29. Goldsworthy MA, Fateen W, Thygesen $\mathrm{H}$, et al. Patient understanding of liver cirrhosis and improvement using multimedia education. Frontline Gastroenterol. 2017;8:214-219.

30. Volk ML, Fisher N, Fontana RJ. Patient knowledge about disease self-management in cirrhosis. Am J Gastroenterol. 2013;108:302-305.

31. Venkatesh V, Davis FD. A model of the antecedents of perceived ease of use: development and test*. Decis Sci. 1996;27:451-481.

32. Bloom P, Marx M, Wang T, et al. A smartphone app is feasible for outpatient cirrhotic ascites management. Iproceedings. 2019;5:e15130.

33. Ertel AE, Kaiser TE, Abbott DE, et al. Use of video-based education and tele-health home monitoring after liver transplantation: Results of a novel pilot study. Surgery. 2016;160:869-876.

34. Bajaj JS, Wade JB, Gibson DP, et al. The multi-dimensional burden of cirrhosis and hepatic encephalopathy on patients and caregivers. Am J Gastroenterol. 2011;106:1646-1653.

35. Bajaj JS, Ellwood M, Ainger T, et al. Mindfulness-based stress reduction therapy improves patient and caregiver-reported outcomes in cirrhosis. Clin Transl Gastroenterol. 2017;8:e108.

36. Shrestha D, Rathi S, Grover S, et al. Factors affecting psychological burden on the informal caregiver of patients with cirrhosis: looking beyond the patient. J Clin Exp Hepatol. 2020;10:9-16.

\section{SUPPORTING INFORMATION}

Additional supporting information may be found online in the Supporting Information section.

How to cite this article: Louissaint J, Lok AS, Fortune BE, Tapper EB. Acceptance and use of a smartphone application in cirrhosis. Liver Int. 2020;40:1556-1563. https://doi. org/10.1111/liv.14494 